\title{
A case of chronic eosinophilic pneumonia in a patient treated with dupilumab
}

This article was published in the following Dove Press journal:

Therapeutics and Clinical Risk Management

\author{
Francesco Menzella' \\ Gloria Montanari ${ }^{1}$ \\ Giulia Patricelli \\ Alberto Cavazza ${ }^{3}$ \\ Carla Galeone \\ Patrizia Ruggiero' \\ Diego Bagnasco 4 \\ Nicola Facciolongo' \\ 'Department of Medical Specialties, \\ Pneumology Unit, Azienda USL di Reggio \\ Emilia, Arcispedale Santa Maria Nuova- \\ IRCCS, Reggio Emilia, Italy; ${ }^{2}$ Pneumology \\ Unit, A. Perrino Hospital, Brindisi, Italy; \\ ${ }^{3}$ Department of Pathology, Azienda USL- \\ IRCCS di Reggio Emilia, Reggio Emilia, \\ Italy; ${ }^{4}$ Allergy \& Respiratory Diseases, \\ University of Genoa, Genoa, Italy
}

\begin{abstract}
The increasing knowledge on inflammatory pathways has driven the development of targeted biological therapies for severe refractory asthma. Among the recently developed biologics, the fully human monoclonal antibody dupilumab is an interesting therapeutic option, given its ability to inhibit the biological effects of both IL-4 and IL-13. We describe the case of a male, Caucasian, 56-year-old patient with allergic and eosinophilic severe asthma. Given the poor asthma control, he started treatment with add-on dupilumab, and after the tenth injection, he presented with a fever and bilateral pulmonary thickening. A significant increase in blood eosinophilia was also reported. The patient underwent a fiberoptic bronchoscopy with bronchoalveolar lavage (BAL) and transbronchial lung biopsy (TBLB/TBB). BAL revealed eosinophils alveolitis (60\%) while TBB showed findings compatible with chronic eosinophilic pneumonia (CEP). After prolonged treatment with oral corticosteroids, the clinical picture improved with resolution of CEP. Since the beginning of dupilumab treatment, simultaneously to a great improvement in asthma control, the patient showed a progressive increase in blood eosinophils count and subsequent onset of clinical-radiological pattern suggestive of CEP. Based on published data, dupilumab may have induced an alteration of the complex immunological pathway of our patient. This pathway is affected by both allergic and eosinophilic asthmatic endotypes, and consequently, the concomitant action of allergenic stimuli and eosinophils may have caused the appearance of eosinophilic pneumonia. To our knowledge, this is the first reported case of CEP as a possible severe side effect of dupilumab administration.
\end{abstract}

Keywords: monoclonal antibodies, dupilumab, severe asthma, eosinophilic pneumonia, eosinophils, fiberoptic bronchoscopy

\section{Introduction}

Severe asthma is a collection of phenotypes and endotypes with several pathophysiological characteristics that result in poor control and frequent exacerbations. Approximately in $50 \%$ of patients with severe refractory asthma, there is strong evidence of the pathogenic role of T helper type-2 (Th2) cytokines, such as IL-5, IL-4 and IL-13, which lead to the eosinophilic and allergic inflammatory processes. ${ }^{1}$ These patients, who are on maintenance treatment with oral corticosteroids (OCS), represent up to 46\% of severe asthma population. ${ }^{2}$ The impact of OCS and their side effects are also very relevant, in terms of quality of life and pharmacoeconomic impact. ${ }^{3}$ Luckily, the increasing knowledge on inflammatory phenotypes has driven the development of targeted biological therapies, especially selective targeted monoclonal antibodies (mAbs).

Among the recently developed anti-asthma biologic drugs, the fully human $\mathrm{mAb}$ dupilumab is an interesting therapeutic option, given its ability to inhibit the biological effects of both IL-4 and IL-13. This drug is directed against the shared IL-4
Correspondence: Francesco Menzella Department of Medical Specialties, Pneumology Unit, Arcispedale Santa Maria Nuova, Azienda USL-IRCCS di

Reggio Emilia, Via Amendola 2, 42I22

Reggio Emilia, Italy

Tel +39052 2296073

Fax +390522296182

Email francesco.menzella@ausl.re.it 
receptor alpha subunit: binding to the receptor, it blocks the signaling pathway of both IL-4 and IL-13, with very promising data in terms of asthma control and reduction in the use of OCS. ${ }^{4}$ The advent of mAbs, starting from omalizumab, has not generated particular concerns about their safety and their long usage, thanks to the accumulation of data on these matters. ${ }^{5}$ On the contrary, the new therapeutic options, including dupilumab, lack of evidence on safety, especially in the long term. Phase III studies have shown that dupilumab, as add-on maintenance therapy, has a significant effect on improving lung function and reducing the rate of asthmatic exacerbations, regardless of baseline blood eosinophil count. ${ }^{6}$ Treatment with dupilumab is particularly useful in patients with severe asthma and associated comorbidities (atopic dermatitis, chronic sinusitis and nasal polyposis), who remain inadequately controlled with usual asthma therapies. ${ }^{6}$ In patients with elevated eosinophil levels and persistent moderate-to-severe asthma, dupilumab therapy was associated with fewer asthma exacerbations when long-acting beta-adrenoceptor agonist (LABA) and inhaled glucocorticoids were withdrawn. ${ }^{7}$ In patients with severe steroid-dependent asthma, dupilumab demonstrated a steroid-sparing action with a simultaneous reduction of severe exacerbations rate and increase of $\mathrm{FEV}_{1} \cdot{ }^{4}$ The recent VENTURE randomized clinical trial (RCT) showed a significant, but transient, blood eosinophilia in the dupilumab group with respect to placebo group (14\% vs $1 \%){ }^{4}$

In this article, we describe the case of a patient with severe refractory asthma treated with dupilumab, who developed early high blood eosinophils levels and finally a chronic eosinophilic pneumonia (CEP). At our best knowledge, this event is the first reported in literature.

\section{Case presentation}

A 56-year-old man, nonsmoker, came to our observation. He was a retired cleaning maintenance technician. Remote clinical history included chronic sinusitis with nasal polyposis, treated with functional endoscopic sinus surgery, a hospitalization in Cardiology Unit in 2011 for a vasospastic angina episode, caused by myocardial bridging without significant stenosis of the remaining coronary tracts. In 2010, a diagnosis of allergic asthma was made. Skin prick tests were positive for house-dust mites, birch and grass and negative for aspergillus fumigatus. Serum precipitins against Aspergillus antigens were negative. Pulmonary function tests showed a severe obstruction $\left(\mathrm{FEV}_{1} 1.95 \mathrm{~L}, 54 \%\right.$ of predicted; $\mathrm{FEV}_{1} / \mathrm{FVC}$ $57 \%$ ) with a complete bronchial reversibility after $400 \mu \mathrm{g}$ of inhaled salbutamol $\left(\mathrm{FEV}_{1} 54 \%\right.$; +16\%). Antineutrophil cytoplasmic antibodies were negative without signs and symptoms compatible with vasculitis. The patient did not have a history of sensitivity to NSAIDs. On February 2016, a chest high-resolution CT scan showed no pleural and parenchymal abnormalities. Total IgE were $406.9 \mathrm{IU} / \mathrm{mL}$, blood eosinophils 660 cells $/ \mathrm{mm}^{3}$ and fractional exhaled nitric oxide (FeNO) value (ppb) 43. Asthma Control Questionnaire-5 (ACQ) score was 3.40, which confirmed poor control of asthma. The pharmacological therapy included formoterol/beclomethasone metered-dose inhaler $100 / 6 \mu \mathrm{g}$, two daily inhalations and as needed (twice a day on an average), tiotropium bromide $2.5 \mu \mathrm{g}$ soft mist inhaler, montelukast $10 \mathrm{mg}$ /day. Montelukast was then suspended because ineffectiveness. Due to frequent exacerbations (on average 5 every 12 months), the patient took at least 4 OCS bursts annually. Given the linger of poor asthma control, with continuous worsening leading to 3 exacerbations in three consecutive months, the patient was enrolled in a phase III RCT on dupilumab in July $2016,{ }^{4}$ with subcutaneous administration of the drug at a dose of $300 \mathrm{mg}$ every 2 weeks. At the third administration of dupilumab, a blood eosinophil value of 1840 cells $/ \mathrm{mm}^{3}$ was detected with an improvement in spirometric data $\left(\mathrm{FEV}_{1} 66 \%\right.$, $+22 \%$ compared to pre-treatment data). FeNO value was 13 ppb. During dupilumab treatment and after the clinical improvement, the patient no longer needed OCS therapy. ACQ-5 score was 0.20, indicative of the marked improvement in asthmatic symptoms. The increase in blood eosinophilia was not alarming because in line with what reported in other patients, and also in light of the clinical and spirometric improvement, we decided to continue with dupilumab treatment. In RCTs was found a much higher percentage of patients with blood eosinophilia in the dupilumab group compared to the placebo group (14\% vs $1 \%)^{4}$

On December 7, 2016, the patient arrived at the Center to perform the 10th injection of dupilumab. The patient reported fever up to $38.5^{\circ} \mathrm{C}$ from about 7 days. Chest $\mathrm{X}$ ray showed pulmonary bilateral opacities (Figure 1), for which the patient was treated with azithromycin $500 \mathrm{mg}$ per day for 6 days.

Due to the persistence of the symptoms, a chest highresolution CT scan was performed, which showed bilateral consolidations with air bronchogram, localized in the upper lobes and the superior lower lobe on the right (Figure 2).

Blood tests showed a further increase in blood eosinophilia $\left(2080\right.$ cells $\left./ \mathrm{mm}^{3}\right)$. For these reasons, it was decided to discontinue dupilumab treatment and the patient was admitted at the Department of Pneumology on December 12, 2016. Upon presentation, his blood pressure was 120/ 


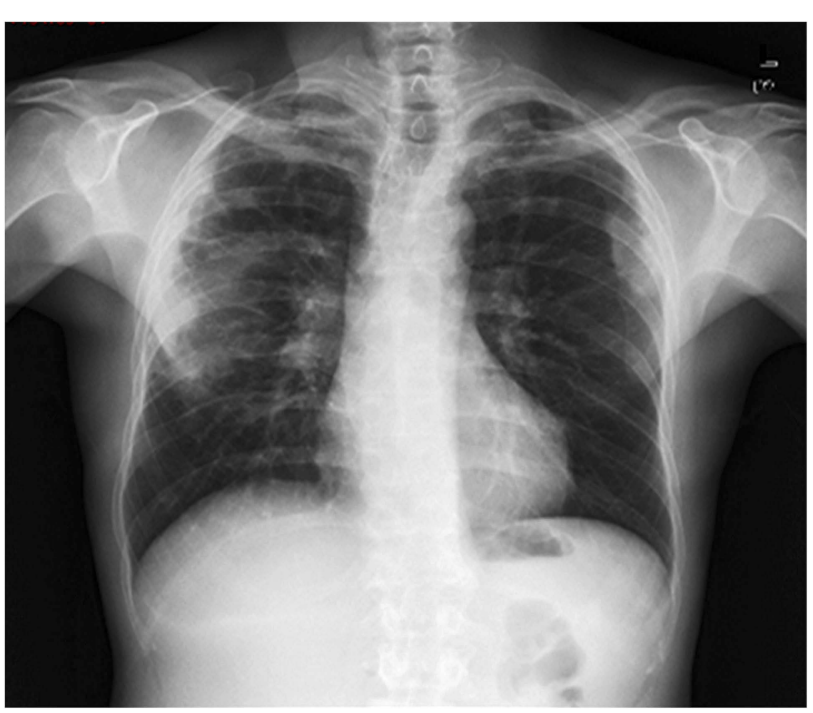

Figure I Chest x-ray: bilateral pulmonary opacities.

$80 \mathrm{mmHg}$, with a heart rate of $84 \mathrm{bpm}$. He was breathing at a rate of $18 \mathrm{bpm}$ and his oxygen saturation was $97 \%$. Complete blood count revealed a white cell count of 10,360 cells $/ \mathrm{mm}^{3}$ with eosinophilic rate of $26.2 \%$. Reactive C protein value was $16.2 \mathrm{mg} / \mathrm{dL}$. Arterial blood gases revealed $\mathrm{pH}$ 7.42; $\mathrm{pO}_{2} 71.4 \mathrm{mmHg} ; \mathrm{pCO}_{2} 35$ $\mathrm{mmHg} ; \mathrm{HCO}_{3} 25.1 \mathrm{mmol} / \mathrm{L}$.

We therefore started an empiric antibiotic treatment (piperacillin/tazobactam $4.5 \mathrm{~g}$ IV every $6 \mathrm{hrs}$ for 10 days and levofloxacin $750 \mathrm{mg}$ once a day for 8 days) without any changes in symptoms. The patient underwent a fiberoptic bronchoscopy with bronchoalveolar lavage (BAL) and transbronchial lung biopsy (TBLB/TBB). BAL revealed eosinophils alveolitis (60\%), whereas TBB showed large fragments of pulmonary parenchyma with inflammatory infiltrate rich in eosinophilic granulocytes, associated with focal aspects of organizing pneumonia and pneumocytes hyperplasia (Figure 3). There were no extravascular granulomas, small and medium-sized vessels vasculitis. The finding was therefore compatible with CEP. A hematological examination was also performed and a search for FIP1L1-PDGFRA, a fusion gene that determines the excessive growth of eosinophils, was recommended. This molecular analysis was negative.

In consideration of the obtained results, we started therapy with prednisolone $50 \mathrm{mg}$ per day for 30 days. Subsequently, a gradual OCS tapering was initiated, and also a rapid clinical improvement and disappearance of fever was noted. The OCS was then suspended after a total of 6 months of therapy and after a new chest CT scan performed in July 2017, confirming resolution of parenchymal thickening. At the end of the OCS therapy, the value of blood eosinophils was 40 cells $/ \mathrm{mm}^{3}$.

\section{Discussion}

The term "eosinophilic lung disease" (ELD) summarizes a group of lung diseases caused by an increase of eosinophils
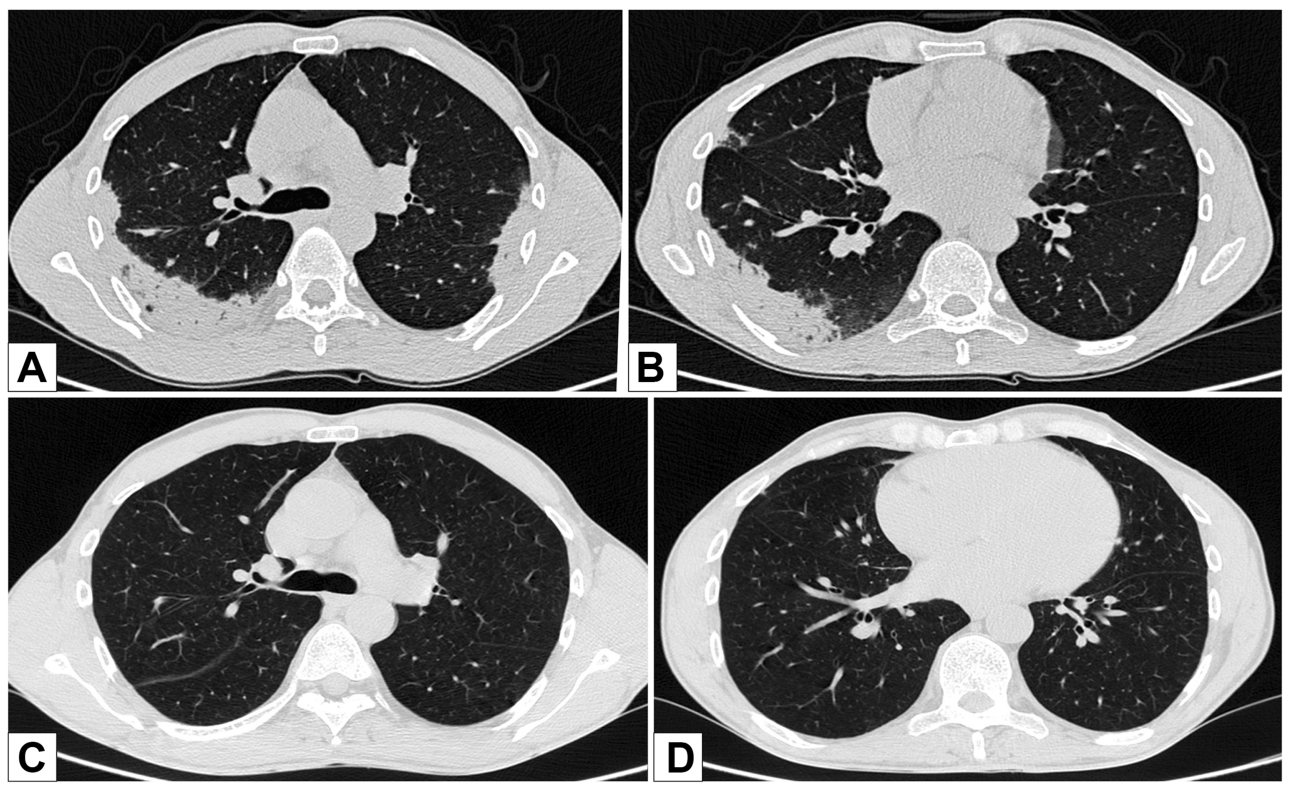

Figure 2 Chest high-resolution CT scan pre-post oral corticosteroids (OCS) treatment: (A) bilaterally, areas of parenchymal thickening with plane bronchograms, confluent, located in the upper lobes bilaterally, and peripherally, in the postero-lateral mantle area of the supero-lateral segment of the upper lobe. (B) Areas of parenchymal thickening with plane bronchograms on the apical of the inferior right lobe. (C, D) Resolution of parenchymal thickening after OCS therapy. 


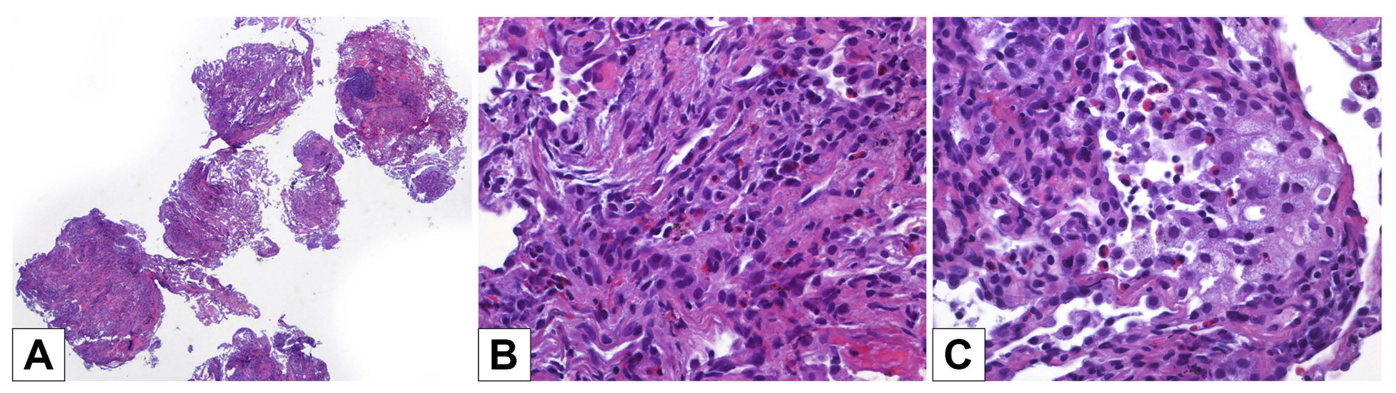

Figure 3 Biopsies: (A) transbronchial biopsies sampled several good-quality lung fragments (hematoxylin-eosin, 20x magnification). (B) A subacute lung injury was present, consisting of foci of organizing pneumonia with pneumocyte hyperplasia and several interstitial and intra-alveolar eosinophils (hematoxylin-eosin, $400 \times$ magnification). (C) In some areas, intra-alveolar foamy macrophages were intermingled with eosinophils (hematoxylin-eosin, $400 \times$ magnification). There were no extravascular granulomas, small and medium-sized vessels vasculitis.

within the lung parenchyma. ELD can be diagnosed in the presence of thoracic opacifications associated with blood eosinophilia, parenchymal eosinophilia confirmed by pulmonary biopsy and important eosinophilia confirmed by BAL. They are divided into diseases with known cause (parasitic infection, allergic bronchopulmonary aspergillosis or drug toxicity), with unknown cause (Loeffler syndrome, acute (AEP) and CEP or idiopathic hypereosinophilic syndrome), and eosinophilic vasculitis (eosinophilic granulomatosis with polyangiitis previously known as Churg-Strauss syndrome) ${ }^{8}$

Among ELD, eosinophilic pneumonia (EP) is an uncommon entity with respiratory symptoms such as dyspnoea and cough, lasting more than 2 weeks. Up to $50 \%$ of patients with EP have a history of asthma. AEP and CEP are usually associated with alveolar eosinophilia $40 \%$ or greater on BAL and usually blood hyper-eosinophilia. ${ }^{9}$ Radiologically EP is characterized by unilateral or bilateral opacities generally with no segmental distribution, often localized in the upper lobes without basilar involvement. ${ }^{10}$ Diagnosis often requires $\mathrm{BAL}$ and/or TBB to confirm respiratory eosinophilia and to exclude other entities, such as infection or malignancy.

In addition to anti-IL-5 Mabs, dupilumab also showed efficacy in patients with severe refractory eosinophilic asthma, regardless of the level of basal blood eosinophilia., ${ }^{4,11}$ In our patient, we found high blood eosinophilia, radiological bilateral consolidation with aerial bronchogram localized in the upper lobes and lower lobe of the right lung. Significant eosinophilia in BAL was also detected, and histological specimens obtained by TBB confirmed the diagnosis of CEP. It is not possible to define with certainty the causes of CEP. As described above, the patient was affected by severe refractory eosinophilic and allergic asthma, and for this reason, he had undergone therapy with dupilumab. Since the beginning of this treatment, simultaneously to a great improvement in asthma control, there was a progressive increase in blood eosinophils count and subsequent onset of clinical-radiological pattern suggestive of ELD/CEP (Figure 4). Although we are not able to establish a certain cause-effect relationship between dupilumab and CEP, it is known that airway eosinophilopoietic processes are modulated by type 2 cytokines such as IL-4 and IL-13, which have been shown to trigger the migratory responses of the hematopoietic stem and progenitor cells (HSPCs) to the airways in asthmatic subjects. ${ }^{12}$ In these processes, other cytokines such as IL-33 are involved. This could promote systemic and local eosinophilic inflammation also by inducing IL-5 production. ${ }^{13}$ From recent data, it seems that HSPCs have an even more complex role through interaction with the alarmins TSLP, IL-33 and IL-25. ${ }^{12}$ In fact, HSPCs express the thymic stromal lymphopoietin receptor and the IL-33 receptor (IL1RL1/ST2). Stimulation of HSPCs with TSLP results in a dose-dependent release of several cytokines, such as IL-5, IL-13, GM-CSF, C-C chemokine (CCL) 22, thymus and activation regulation chemokine, IL-8 and CCL1. ${ }^{14}$ The priming effects of TSLP and IL-33 are inhibited in the presence of the respective receptor blocking antibodies, in particular the IL-13Ral antibody. ${ }^{14}$ Alarmins induce the synthesis of IL-4 and IL-13 from HPC, well-known priming agents of the HSPCs themselves. It has therefore been hypothesized that alarmins directly stimulate the migration of HSPCs with an IL-13dependent mechanism, which can therefore be modified by pharmacological interactions, such as the ones with dupilumab. ${ }^{15}$

Eosinophils represent the largest IL-4-competent population in steady-state allergic tissues and in type 2 inflammatory contexts. ${ }^{16-18}$ It is also known that the production 


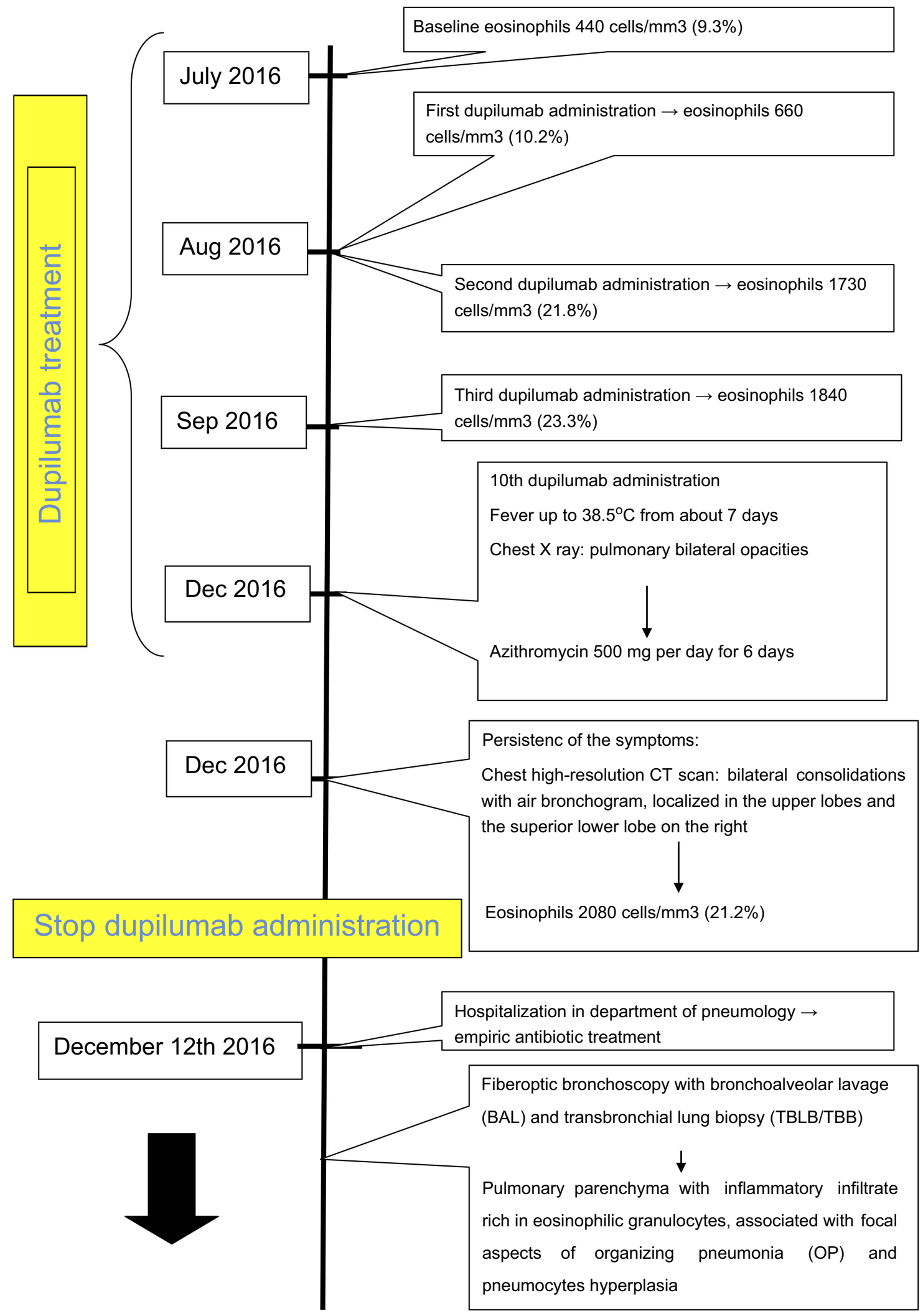

Prednisolone $50 \mathrm{mg}$ per day for 30 days $\rightarrow$ gradual OCS tapering with rapid clinical improvement and disappearance of fever

Figure 4 Case report timeline. 
of IL-4 by eosinophils is extremely important in the regulation of multiple metabolic pathways. ${ }^{19}$ Consequently, in addition to stimulating IL-5 production, the role of IL-4 and IL-13 in the "T2-high" inflammatory pathway should be considered central. Notionally, Webb et al., highlighted the interaction between these three cytokines and observed that the levels of IL-5 and blood eosinophilia produced in the absence of IL-4 are significantly greater in IL13-/mice than in wild-type mice. In addition, it seemed that low levels of IL-13 may result in the activation of NF-kB and an IL-4-independent mechanism of IL-5 production and eosinophil mobilization, suggesting alternative mechanisms of eosinophils activation which should be further studied. ${ }^{20,21}$

Based on previously published data, ${ }^{22}$ the dupilumabinduced IL-4 and IL-13 receptor blockade may have resulted in an alteration of the complex immune-allergic pathway in our patient. This pathway is affected by both allergic and eosinophilic asthmatic endotypes, and consequently, the concomitant action of allergenic stimuli and eosinophils, mediated by a large number of important cytokines including not only Il4 and IL-13, but also alarmins and IL-5, could have caused the appearance of eosinophilic pneumonia during treatment with dupilumab. Another possibility is the correlation with antibiotic therapy. However, eosinophils levels were high before the antibiotic treatment beginning, the pulmonary thickening was already present, and there were no signs of purulent sputum or cough and therefore it seems unlikely to be induced by the azithromycin. As a last hypothesis, it is also possible, though less likely, that the withdrawal of OCS, as a consequence of the steroid-sparing effect of dupilumab, has evidenced the presence of CEP, which was not secondary to the action of the dupilumab itself.

\section{Conclusion}

To our knowledge, this is the first reported case of CEP as a possible severe side effect of dupilumab administration. Nowadays, dupilumab seems to be generally well tolerated, but large-scale studies are required to establish its long-term safety and tolerability. We suggest a close monitoring of blood eosinophils count and clinical parameters in patients with severe refractory asthma treated with this $\mathrm{mAb}$, which remains a very promising and effective therapeutic option.

\section{Consent for publication}

Written informed consent was obtained from the patient for publication of this case report and accompanying images. Institutional approval is not required to publish the case details.

\section{Disclosure}

Francesco Menzella participated in contracted research and clinical trials for Novartis and Sanofi; has received lecture fees and advisory board fees from AstraZeneca, Boehringer Ingelheim, Chiesi, GlaxoSmithKline, Mundipharma and Novartis. Nicola Facciolongo served as a consultant for Boston Scientific and has received lecture fees from AstraZeneca and Chiesi. The other authors report no conflicts of interest in this work.

\section{References}

1. Loza MJ, Djukanovic R, Chung KF, et al. ADEPT (Airways disease endotyping for personalized therapeutics) and U-BIOPRED (Unbiased biomarkers for the prediction of respiratory disease outcome consortium) investigators. Validated and longitudinally stable asthma phenotypes based on cluster analysis of the ADEPT study. Respir Res. 2016;17(1):165.

2. Shaw DE, Sousa AR, Fowler SJ, et al. Clinical and inflammatory characteristic of the European U-BIOPRED adult severe asthma cohort. Eur Respir J. 2015;46(5):1308-1321. doi:10.1183/13993003.00779-2015

3. Lefebvre P, Duh MS, Lafeuille MH, et al. Acute and chronic systemic corticosteroid-related complications in patients with severe asthma. $J$ Allergy Clin Immunol. 2015;136(6):1488-1495. doi:10.1016/j.jaci. 2015.07.046

4. Rabe KF, Nair P, Brusselle G, et al. Efficacy and safety of dupilumab in glucocorticoid-dependent severe asthma. N Engl J Med. 2018;378 (26):2475-2485. doi:10.1056/NEJMoa1804093

5. Adachi M, Kozawa M, Yoshisue H, et al. Real-world safety and efficacy of omalizumab in patients with severe allergic asthma: a long-term post-marketing study in Japan. Respir Med. 2018;141:5663. doi:10.1016/j.rmed.2018.06.021

6. Barranco P, Phillips-Angles E, Dominguez-Ortega J, Quirce S. Dupilumab in the management of moderate-to-severe asthma: the data so far. Ther Clin Risk Manag. 2017;13:1139-1149. doi:10.214 7/TCRM.S125964

7. Wenzel S, Ford L, Pearlman D, et al. Dupilumab in persistent asthma with elevated eosinophil levels. N Engl J Med. 2013;368(26):24552466. doi: $10.1056 /$ NEJMoa1304048

8. Weissler JC. Eosinophilic lung disease. Am J Med Sci. 2017;354 (4):339-349. doi:10.1016/j.amjms.2017.03.020

9. Bartal C, Sagy I, Barski L. Drug-induced eosinophilic pneumonia: a review of 196 case reports. Medicine (Baltimore). 2018;97(4):e9688. doi:10.1097/MD.0000000000009688

10. Price M, Gilman MD, Carter BW, Sabloff BS, Truong MT, Wu CC. Imaging of eosinophilic lung diseases. Radiol Clin North Am. 2016;54(6):1151-1164. doi:10.1016/j.rcl.2016.05.008

11. Castro M, Corren J, Pavord ID, et al. Dupilumab efficacy and safety in moderate-to-severe uncontrolled asthma. $N$ Engl J Med. 2018;378 (26):2486-2496. doi:10.1056/NEJMoa1804092

12. Punia N, Smith S, Thomson JV, Irshad A, Nair P, Sehmi R. Interleukin-4 and interleukin-13 prime migrational responses of haemopoietic progenitor cells to stromal cell-derived factor-1 $\alpha$. Clin Exp Allergy. 2012;42(2):255-264. doi:10.1111/j.1365-2222.2011.03889.x

13. Johnston LK, Hsu CL, Krier-Burris RA, et al. IL-33 precedes IL-5 in regulating eosinophil commitment and is required for eosinophil homeostasis. J Immunol. 2016;197:3445-3453. doi:10.4049/jimmunol.160 0611 
14. Allakhverdi Z, Comeau MR, Smith DE, et al. CD34p hemopoietic progenitor cells are potent effectors of allergic inflammation. $J$ Allergy Clin Immunol. 2009;123:472-478. doi:10.1016/j.jaci.2 008.10 .022

15. Smith SG, Gugilla A, Mukherjee M, et al. Thymic stromal lymphopoietin and IL-33 modulate migration of hematopoietic progenitor cells in patients with allergic asthma. J Allergy Clin Immunol. 2015;135:1594-1602. doi:10.1016/j.jaci.2014.12.1918

16. Phillips C, Coward WR, Pritchard DI, Hewitt CR. Basophils express a type 2 cytokine profile on exposure to proteases from helminths and house dust mites. J Leukoc Biol. 2003;73:165-171. doi:10.1189/ jlb.0702356

17. Wu D, Molofsky AB, Liang HE, et al. Eosinophils sustain adipose alternatively activated macrophages associated with glucose homeostasis. Science. 2011;332:243-247. doi:10.1126/science.332.6026. $173-\mathrm{c}$
18. Shinkai K, Mohrs M, Locksley RM. Helper T cells regulate type-2 innate immunity in vivo. Nature. 2002;420:825-829. doi:10.1038/ nature 01131

19. Qiu Y, Nguyen KD, Odegaard JI, et al. Eosinophils and type 2 cytokine signaling in macrophages orchestrate development of functional beige fat. Cell. 2014;157:1292-1308. doi:10.1016/j. cell.2014.03.040

20. Webb DC, McKenzie AN, Koskinen AM, Yang M, Mattes J, Foster PS. Integrated signals between IL-13, IL-4, and IL-5 regulate airways hyperreactivity. J Immunol. 2000;165(1):108-113. doi:10.4049/ jimmunol.165.1.108

21. Gour N, Wills-Karp M. IL-4 and IL-13 signaling in allergic airway disease. Cytokine. 2015;75(1):68-78. doi:10.1016/j.cyto.2015.05.014

22. Gandhi NA, Pirozzi G, Graham NMH. Commonality of the IL-4/IL13 pathway in atopic diseases. Expert Rev Clin Immunol. 2017;13 (5):425-437. doi:10.1080/1744666X.2017.1298443

\section{Publish your work in this journal}

Therapeutics and Clinical Risk Management is an international, peerreviewed journal of clinical therapeutics and risk management, focusing on concise rapid reporting of clinical studies in all therapeutic areas, outcomes, safety, and programs for the effective, safe, and sustained use of medicines. This journal is indexed on PubMed Central, CAS,
EMBase, Scopus and the Elsevier Bibliographic databases. The manuscript management system is completely online and includes a very quick and fair peer-review system, which is all easy to use. Visit http://www.dovepress.com/testimonials.php to read real quotes from published authors. 